\title{
An Exact Earth-Flattening Procedure in Propagation Around a Sphere
}

\author{
Benjamin Y.-C. Koo ${ }^{2}$ and Martin Katzin ${ }^{2}$
}

(August 17, 1959)

\begin{abstract}
By a refinement of the procedure used in the usual earth-flattening approximation, the problem of propagation around a spherical earth is reduced to an exact equation of the same form. Thereby the earth-flattening procedure becomes applicable to arbitrarily large heights and distances. It is also found that existing solutions of the approximate equations can be re-evaluated to yield the exact solutions for slightly different refractive index distributions.
\end{abstract}

\section{Introduction}

The problem of propagation of radio waves around a spherical earth has been attacked by many writers over a period of more than a half a century. For the idealized case of homogeneous atmosphere and earth, the presently accepted solution is due to van der Pol and Bremmer [1]. This makes use of a modified form of the Watson transformation to convert from a very slowly converging series of spherical harmonics to a more rapidly converging series of residue waves. Physically, the transformation is from waves progressing radially out from the earth to waves which progress along the earth's surface.

In order to reduce the mathematical complexities accompanying the van der Pol-Bremmer treatment, M. H. L. Pryce $[2,3]$ introduced the so-called earthflattening approximation, in which plausible approximations are introduced to simplify the original differential equations. This procedure reduces the problem of propagation over spherical earth to propagation over a plane earth with an atmosphere having a modified refractive index. In this way the van der Pol-Bremmer type of result for the homogeneous atmosphere can be obtained in a much more direct manner. Furthermore, the method can be applied to inhomogeneous atmospheres in which the refractive index varies only with height (so-called horizontal stratification).

Pekeris [4] investigated the accuracy of the earthflattening approximation in the case of the standard atmosphere (in which the modified refractive index increases linearly with height). He found that the accuracy decreased with increasing distance along the earth's surface and also with increasing height above the surface. If $r$ is the great-circle distance and $a$ the earth's radius, the error with distance is proportional to $(r / a)^{2}$ independently of the frequency, amounting to about 8 percent at $r / a=1$. The error

\footnotetext{
1 The research reported in this paper was supported in part by the Missile and Space Vehicles Department of the General Electric Co.

2 Electromagnetic Research Corp., Washington, D.C.
}

with height, however, is proportional to frequency, and to height as $h^{5 / 2}$. The height error becomes serious in the microwave region at heights in the order of a few thousand feet.

In this paper we improve and extend the Pryce and Pekeris treatments to obtain exact differential equations for the spherical geometry in terms of equations of plane earth type. The solutions of these hold for arbitrary heights and distances. Comparison with the previous earth-flattening approximation leads to an interpretation of the errors therein.

\section{Formulation of the Problem}

We consider propagation from a source above a spherical earth of radius $a$ with an atmosphere whose refractive index $n$ is a function only of the radial distance (i.e., horizontally-stratified atmosphere). The treatment is simplified without any essential restriction by assuming that radiation from the source is independent of azimuth (we shall indicate later the extension to remove this restriction). Then it can be shown [5] that the electric and magnetic fields at any point may be derived from a radial Hertz vector $\psi$, which satisfies the reduced wave equation

$$
\nabla^{2} \psi+k_{0}^{2} n^{2} \psi=0
$$

where $k_{0}=2 \pi / \lambda=2 \pi f / c$. By adopting a spherical coordinate system $(R, \theta, \phi)$ with origin at the center of the earth and with the source on the polar axis, (2.1) (with the assumed azimuth independence of $\psi$ ) reduces to

$$
\frac{1}{R^{2}} \frac{\partial}{\partial R}\left(R^{2} \frac{\partial \psi}{\partial R}\right)+\frac{1}{R^{2} \sin \theta} \frac{\partial}{\partial \theta}\left(\sin \theta \frac{\partial \psi}{\partial \theta}\right)+k_{0}^{2} n^{2} \psi=0 .
$$

This equation can be separated in the usual way by letting

$$
\psi=T(\theta) U(R),
$$


whereby there result the two ordinary differential equations

$$
\begin{gathered}
\frac{d^{2} U}{d R^{2}}+\frac{2}{R} \frac{d U}{d R}+k_{0}^{2}\left[n^{2}-\left(\frac{a S}{R}\right)^{2}\right] U=0, \\
\frac{d^{2} T}{d \theta^{2}}+\cot \theta \frac{d T}{d \theta}+(a s)^{2} T=0,
\end{gathered}
$$

in which $(a s)^{2}$ is the separation constant and $S=s / k_{0}$.

We now introduce the transformations ${ }^{3}$

$$
\begin{aligned}
\eta & =a \log (R / a), \\
r & =a \theta .
\end{aligned}
$$

Equations (2.3) and (2.4) become, respectively,

$$
\begin{aligned}
& \frac{d^{2} U}{d \eta^{2}}+\frac{1}{a} \frac{d U}{d \eta}+k_{0}^{2}\left(n^{2} e^{2 \eta / a}-S^{2}\right) U=0, \\
& \frac{d^{2} T}{d r^{2}}+\frac{1}{a} \cot (r / a) \frac{d T}{d r}+s^{2} T=0 .
\end{aligned}
$$

$S^{2}$ is the eigenvalue of the differential eq (2.7), which eventually is determined by the boundary conditions.

The problem of solving (2.1) thus is equivalent to solving (2.7) and (2.8).

\section{The Height-Gain Function U}

We consider first (2.7) for the herght-gain function $U$. By introducing a new variable $V$, given by

$$
U=(a / R)^{\frac{1}{2}} V=V e^{-\eta /(2 a)},
$$

(2.7) becomes

$$
\frac{d^{2} V}{d \eta^{2}}+k_{0}^{2}\left(N^{2}-\sigma^{2}\right) V=0
$$

in which we introduce as our definition of the modified refractive index

$$
N=n e^{\eta / a}=n R / a,
$$

and define

$$
\sigma^{2}=S^{2}+1 /\left(2 k_{0} a\right)^{2} .
$$

(3.2) is exactly the same in form as the plane-earth equation, which is

$$
\frac{d^{2} U_{0}}{d h^{2}}+k_{0}^{2}\left(\bar{N}^{2}-S^{2}\right) U_{0}=0,
$$

where $h=R-a$ is the actual height above the earth's surface, and

$$
\vec{N}^{2}=n^{2}(1+h / a)^{2} \approx n^{2}(1+2 h / a) .
$$

\footnotetext{
3 These are the transformations used by Pryce [3], who credited Pekeris for the
} range transformation and $\mathrm{E}$. T. Copson for the height transformation.
The method of the usual earth-flattening approximation is to approximate $U$ by $U_{0}$, which is the solution of (3.3). But $U$ can be obtained exactly by means of (3.1), in which $V$ is the solution of (3.2). This fact will permit us to interpret properly the significance of the earth-flattening approximation with respect to the height dependence.

\section{Interpretation of the Earth-Flattening Approximation}

Equations (3.2) and (3.3) are identical only in form. However, the height variable $\eta$ differs from $h$, and, for a given atmosphere, $N$ as a function of $\eta$ will not have the same analytical form as $\bar{N}$ as a function of $h$. In addition, the eigenvalue $\sigma^{2}$ differs from $S^{2}$ by the small real quantity $1 /\left(2 k_{0} a\right)^{2}$. Thus it follows that a given solution $U_{0}$ of the approximate earth-flattened eq (3.3) corresponds (but is not equal) to the exact solution of a slightly different problem. The approximate solution differs from the corresponding exact solution in three respects, namely: (a) $\mathrm{By}$ the factor $(a / R)^{1 / 2}$ in (3.1) (this factor usually is nearly unity); (b) the modified index profile is different because of the difference in height scale; ${ }^{4}$ (c) the eigenvalue is shifted slightly. Both (b) and (c) lead to differences between $U_{0}$ and $U$ which involve the frequency. (b) probably is the more important effect, so that the error of the earthflattening approximation as a function of height probably is due primarily to the choice of height scale.

\section{The Standard Atmosphere}

The standard atmosphere is now defined by

$$
\begin{aligned}
\bar{N}^{2}(h) & =1+q h, \\
q & =3 /(2 a) .
\end{aligned}
$$

In this case, (3.3) becomes Stokes' equation, whose solutions are Airy functions, or the modified Hankel functions of order one-third [6]. In view of the fact that an exact solution is available in terms of our function $U$, it appears that it mav be desirable to define the standard atmosphere by the relation

$$
N^{2}(\eta)=1+q \eta \text {. }
$$

In this case (3.2) likewise becomes Stokes' equation.

\section{The Distance Function T}

The distance dependence is given by the function $T$, which satısfies the Legendre differential eq (2.8). In the earth-flattening approximation, $T$ is approximated by a function $T_{0}$ which satisfies the differential equation

$$
\frac{d^{2} T_{0}}{d r^{2}}+\frac{1}{r} \frac{d T_{0}}{d r}+s^{2} T_{0}=0
$$

\footnotetext{
${ }_{4}^{4}$ This shall be discussed in greater detail for specific cases in a later paper.
} 
which is the Bessel equation of order zero. Equation (6.1) gives the distance dependence for plane earth.

In investigating the accuracy of this approximation, Pekeris [4] assumed that $T$ could be written in the form

$$
T(r)=T_{0}(r)+(a s)^{-2} T_{2}(r)+(a s)^{-4} T_{4}(r)+\ldots . .
$$

After substituting (6.2) into (2.8), expanding $\cot (r / a)$ in a power series in $r / a$ and equating the coefficients of like powers of $(a s)^{-2}$, he obtained a system of simultaneous differential equations, which is written in terms of the operator

as follows:

$$
L(T)=\left(\frac{d^{2}}{d x^{2}}+\frac{1}{x} \frac{d}{d x}+1\right) T,
$$

$$
\begin{aligned}
& L\left(T_{0}\right)=0, \\
& L\left(T_{2}\right)=\frac{x}{3} \frac{d T_{0}}{d x}, \\
& L\left(T_{4}\right)=\frac{x^{3}}{45} \frac{d T_{0}}{d x}+\frac{x}{3} \frac{d T_{2}}{d x}, \\
& L\left(T_{6}\right)=\frac{2 x^{5}}{945} \frac{d T_{0}}{d x}+\frac{x^{3}}{45} \frac{d T_{2}}{d x}+\frac{x}{3} \frac{d T_{4}}{d x},
\end{aligned}
$$

where $x=s r$.

Pekeris wrote the solutions of (6.3a) and (6.3b) in terms of the Hankel functions (corresponding to an implicit time function $e^{i \omega t}$ )

$$
\begin{aligned}
& T_{0}=H_{0}^{(2)}(s r), \\
& T_{2}=\frac{(s r)^{2}}{12} H_{2}^{(2)}(s r),
\end{aligned}
$$

and used

$$
(a s)^{-2} T_{2}(r)=-(1 / 12)(r / a)^{2} H_{2}^{(2)}(s r)
$$

as the term to estimate the error in using $T_{0}$ as the solution of (2.8).

We now proceed to extend Pekeris' treatment to solve for $T_{2 n}(r)$ for arbitrary $n$. The solution will be obtained in terms of cylinder functions of correspondingly higher order. Hence it is possible to obtain $T(r)$ in the form (6.2) to any desired degree of accuracy for any distance $r$.

Let $Z_{n}(x)$ be any cylinder function of order $n$. Define

and

$$
C_{n}(x) \equiv x^{n} Z_{n}(x)
$$

$$
L(T) \equiv T^{\prime \prime}+x^{-1} T^{\prime}+T,
$$

where primes indicate derivatives with respect to $x$. Then $C_{n}$ satisfies the differential equation.

$$
L\left(C_{n}\right)=(2 n / x) C_{n}^{\prime} .
$$

Now a solution of $(6.3 \mathrm{a})$ is

and since

$$
T_{0}=C_{0}=Z_{0},
$$

(6.3b) becomes

$$
C_{0}^{\prime}=-x^{-2} C_{2}^{\prime} \text {, }
$$

$$
L\left(T_{2}\right)=-(1 / 3) x^{-1} C_{2}^{\prime}=-(1 / 12) 4 x^{-1} C_{2}^{\prime} .
$$

Hence from (6.4) it follows that

$$
T_{2}=-(1 / 12) C_{2}=-(1 / 12)(s r)^{2} Z_{2}(s r) .
$$

Similar procedures may be applied to $(6.3 \mathrm{c}),(6.3 \mathrm{~d})$, . . by applying the following reduction formula to reduce the terms on the right-hand side to a sum of terms of the form $2 n x^{-1} C_{n}^{?}$ :

$$
x^{2 k} C_{m}^{\prime}=\sum_{\alpha=0}^{k} C_{\alpha, k, m} C_{m+k+\alpha,}^{\prime} \quad k=1,2, \ldots,
$$

where

$$
\left.\begin{array}{l}
C_{0, k, 0}=0, \\
C_{\alpha, k, m}=(-1) \frac{\alpha k !(m+k-1) ! 2^{k-\alpha}}{\alpha !(k-\alpha) !(m+\alpha-1) !}, \quad m+\alpha \neq 0 .
\end{array}\right\}
$$

Equation (6.6) may be derived from the recurrence formulas for the cylinder functions. The solutions for $T_{4}$ and $T_{6}$ are given below:

$$
\begin{aligned}
& T_{4}=-\frac{1}{30} C_{3}+\frac{1}{160} C_{4}, \\
& T_{6}=-\frac{17}{840} C_{4}+\frac{19}{2520} C_{5}-\frac{61}{120,960} C_{6} .
\end{aligned}
$$

In most cases of propagation around the earth, one or two terms of (6.2) give sufficient accuracy.

Finally, the treatment may be extended in a similar way to the case of $\partial \psi / \partial \phi \neq 0$. Equation (2.4) then is replaced by the associated Legendre equation, for which we have obtained a solution in terms of generalized $C$ functions

$$
C_{n, m}(x)=x^{n} Z_{m+n}(x) .
$$

The details of this development will be reported elsewhere.

\section{Conclusions}

By a refinement of the procedure used in the usual earth-flattening approximation, we have shown that the problem of propagation around a spherical earth can be reduced to an exact equation of the same form. Thereby the earth-flattening procedure becomes applicable to arbitrarily large heights and distances. It is also found that existing solutions of the approximate equations can be re-evaluated to yield the exact solutions for slightly different refractive index distributions. 


\section{References}

[1] B. van der Pol and H. Bremmer, The diffraction of electromagnetic waves from an electrical point source round a finitely conducting sphere, with applications to radiotelegraphy and the theory of the rainbow, Phil. Mag. 24, 141, 825 (Suppl.) (1937); The propagation of radio waves over a finitely conducting spherical earth, Phil. Mag. 25, 817 (Suppl.) (1938); Further note on the propagation of radio waves over a finitely conducting spherical earth, Phil. Mag. 27, 261 (1939).

[2] M. H. L. Pryce (unpublished).

[3] M. H. L. Pryce, The diffraction of radio waves by the curvature of the earth, Advances in Phys. 2, 67 (1953).
[4] C. L. Pekeris, Accuracy of the earth-flattening approximation in the theory of microwave propagation, Phys. Rev. \%0, 518 (1946).

[5] H. Bremmer, Terrestrial radio waves, p. 139 (Elsevier Publishing Co., Inc., Amsterdam, Netherlands, 1949).

[6] Computation La oratory, Harvard University, Tables of the modified Hankel functions of order one-third and of their derivatives (Harvard University Press, Cambridge, Massachusetts, 1945).

Boulder, Colo.

(Paper 64D1-39) 